\title{
Modeling aerotaxis band formation in Azospirillum brasilense
}

\author{
Mustafa Elmas ${ }^{1}$, Vasilios Alexiades ${ }^{1}$, Lindsey $\mathrm{O}^{\prime} \mathrm{Neal}^{2}$ and Gladys Alexandre ${ }^{2^{*}}$ (D)
}

\begin{abstract}
Background: Bacterial chemotaxis, the ability of motile bacteria to navigate gradients of chemicals, plays key roles in the establishment of various plant-microbe associations, including those that benefit plant growth and crop productivity. The motile soil bacterium Azospirillum brasilense colonizes the rhizosphere and promotes the growth of diverse plants across a range of environments. Aerotaxis, or the ability to navigate oxygen gradients, is a widespread behavior in bacteria. It is one of the strongest behavioral responses in A. brasilense and it is essential for successful colonization of the root surface. Oxygen is one of the limiting nutrients in the rhizosphere where density and activity of organisms are greatest. The aerotaxis response of A. brasilense is also characterized by high precision with motile cells able to detect narrow regions in a gradient where the oxygen concentration is low enough to support their microaerobic lifestyle and metabolism.

Results: Here, we present a mathematical model for aerotaxis band formation that captures most critical features of aerotaxis in A. brasilense. Remarkably, this model recapitulates experimental observations of the formation of a stable aerotactic band within 2 minutes of exposure to the air gradient that were not captured in previous modeling efforts. Using experimentally determined parameters, the mathematical model reproduced an aerotactic band at a distance from the meniscus and with a width that matched the experimental observation.

Conclusions: Including experimentally determined parameter values allowed us to validate a mathematical model for aerotactic band formation in spatial gradients that recapitulates the spatiotemporal stability of the band and its position in the gradient as well as its overall width. This validated model also allowed us to capture the range of oxygen concentrations the bacteria prefer during aerotaxis, and to estimate the effect of parameter values (e.g. oxygen consumption rate), both of which are difficult to obtain in experiments.
\end{abstract}

Keywords: Chemotaxis, Aerotaxis, Band formation, Azospirillum brasilense, Mathematical modeling

\section{Background}

Plant-microbe associations play a vital role in plant health and crop productivity. The ability to detect and respond to environmental changes in the vicinity of bacteria is essential for their survival and growth. A variety of mechanisms have evolved by which cells sense their environmental changes and respond appropriately. One of the best characterized bacterial responses to changes in the environment is chemotaxis, the ability of motile cells to navigate chemical gradients [1]. In chemotaxis, motile bacteria efficiently and rapidly respond to changes in

*Correspondence: galexan2@utk.edu

${ }^{2}$ Biochemistry and Cellular \& Molecular Biology, University of Tennessee, 1311 Cumberland Ave, Knoxville, TN, 37996 USA

Full list of author information is available at the end of the article the chemical composition of their environment, moving towards regions with increasing concentrations of favorable chemicals (chemoattractants) and away from regions with increasing concentration of unfavorable chemicals (chemorepellents) by biasing their basal motility pattern. Motility and the ability of bacteria to locate niches that support optimum growth in the rhizosphere by chemotaxis is essential for their survival and enhances their competitiveness in this environment $[2,3]$.

Aerotaxis is chemotaxis in an oxygen gradient. This bacterial behavior was first reported by Engelmann in 1881 . He observed the aggregation of an organism around air bubbles [4, 5]. Beijerinck later confirmed Engelmann's finding and further described the formation of a sharp 
band of motile cells, corresponding to their accumulation, around a source of oxygen [6]. He also observed that the band of motile organisms descended when air was replaced with oxygen and ascended when air was replaced with hydrogen, implying that the organisms moved towards a specific concentration of oxygen. The preferred concentration of oxygen in a gradient has been determined for a few motile bacterial species (reviewed in [2]) including $200 \mu \mathrm{M}$ for Bacillus subtilis (an obligate aerobe), $50 \mu \mathrm{M}$ for Escherichia coli (a facultative anaerobe), $0.4 \mu \mathrm{M}$ for Desulfovibrio vulgaris (an aerotolerant anaerobe), and 3-5 $\mu \mathrm{M}$ for Azospirillum brasilense (a microaerobe).

There are two types of aerotaxis responses known to date. In aerobes such as $B$. subtilis motile bacteria respond directly to the oxygen concentration and accumulate at the highest concentrations of oxygen in the gradient [7]. In other organisms, such as $E$. coli and A. brasilense, cells perform aerotaxis not by sensing oxygen itself, but by monitoring the effects that oxygen has on the metabolism of the cells $[8,9]$. This behavior is broadly referred to as energy taxis [2]. In energy taxis-based aerotaxis, cells do not move toward the greatest concentration of oxygen but toward an intermediate concentration of oxygen that supports maximum energy levels. The signal for this type of behavior originates within the electron transport system, where oxygen-mediated changes of the rate of electron transport, redox status or proton motive force are detected during aerotaxis $[2,10]$.

Aerotaxis is a major behavioral response in $A$. brasilense [11], characterized by a remarkable ability to precisely locate niches where oxygen concentrations are low and optimal to support metabolism. At such locations motile cells form sharp bands (as seen in Fig. 1). A. brasilense senses very high and low oxygen concentrations as repellents and accumulates at intermediate concentrations, preferring about $5 \mu \mathrm{M}$ dissolved oxygen [10] (note that $1 \%$ of oxygen in air corresponds to 13 $\mu \mathrm{M}$ dissolved oxygen in water). Energy taxis guides $A$. brasilense to move towards microenvironments optimal for maximum energy generation and nitrogen fixation $[10,12]$. The location and width of a band are primary observable and measurable quantities in aerotaxis experiments [13].

The motile soil bacterium Azospirillum brasilense colonizes the rhizosphere and promotes the growth of a variety of plants across a range of environments. It is 2-3 $\mu \mathrm{m}$ long, with a single polar flagellum, [14]. When the flagellum rotates counterclockwise (CCW), the cell moves forward on a straight line, called a run. When the flagellum rotates clockwise $(\mathrm{CW})$, the cell moves backward, and may also change direction, called a reversal. The frequency of reversals determines whether the cell moves away (when reversal frequency is low, so runs predominate) or remains nearby (when reversal frequency is high).

A model of aerotactic band formation, incorporating energy taxis, was developed by Mazzag et al. [15]. It is based on earlier models for chemotaxis and aerotaxis, which consider the movement of bacteria in one dimension and distinguishes right- and left-moving cells depending on their swimming direction to the oxygen gradient. While the model [15] captured some of the features of the aerotaxis response of $A$. brasilense, it failed to produce a stable aerotactic band, which is typical of that formed by $A$. brasilense.

Here, we use the same basic energy taxis model of Mazzag et al., with some adjustments, enhanced numerical implementation, and use experimentally measured parameter values for $A$. brasilense (Table 1 ), to recapitulate the aerotactic band formation in this species (Fig. 2). We also present numerical simulations to predict how the $A$. brasilense aerotaxis band would respond to changes in parameters (Table 2). Details on implementation and differences with [15] are given at the end of Mathematical Model section.

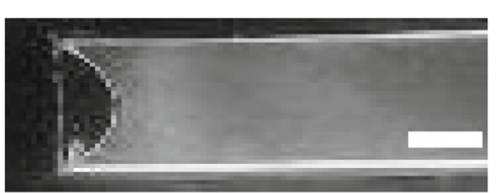

a

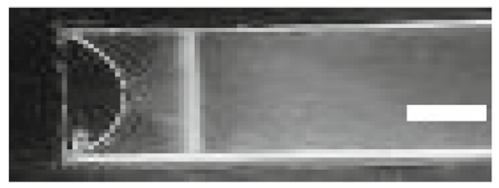

C

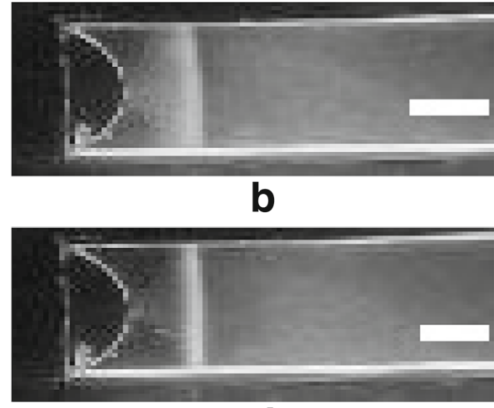

d

Fig. 1 Images of aerotactic band formation of wild-type (Sp7) A. brasilense with 21\% oxygen set at the meniscus. (a) At time 0 sec, when oxygen is applied at the meniscus. (b) At time $50 \mathrm{sec}$. (c) At time $100 \mathrm{sec}$. (d) At time $140 \mathrm{sec}$, by which time the band has already stabilized. Scale bar is $500 \mu \mathrm{m}$ in all panels 
Table 1 Parameter values for the aerotactic band formation model

\begin{tabular}{|c|c|c|}
\hline Parameter & Description & Value \\
\hline$B_{0}$ & Total bacteria concentration & $7 \times 10^{8} \mathrm{cells} / \mathrm{ml}$ \\
\hline$C_{0}$ & $\begin{array}{l}\text { Oxygen concentration at the } \\
\text { meniscus }\end{array}$ & $21 \%$ \\
\hline D & Oxygen Diffusion coefficient & $2 \times 10^{3} \mu \mathrm{m}^{2} / \mathrm{s}$ \\
\hline K & $\begin{array}{l}\text { Bacteria oxygen consumption } \\
\text { rate }\end{array}$ & $4 \times 10^{-9} \mu \mathrm{M} \mathrm{ml} / \mathrm{s}$ cell \\
\hline$v$ & Swimming speed & $20 \mu \mathrm{m} / \mathrm{s}$ \\
\hline$F_{\text {max,band }}$ & $\begin{array}{l}\text { maximum reversal frequency } \\
\text { inside the band }\end{array}$ & $0.96 / \mathrm{s}$ \\
\hline$F_{\max }$ & $\begin{array}{l}\text { maximum reversal frequency } \\
\text { outside of band }\end{array}$ & $0.65 / \mathrm{s}$ \\
\hline$F_{\min }$ & minimum reversal frequency & $0.35 / \mathrm{s}$ \\
\hline$\widehat{C}_{\max }$ & $\begin{array}{l}\text { Upper detectable oxygen } \\
\text { concentration }\end{array}$ & $10 \%$ \\
\hline$C_{\max }$ & $\begin{array}{l}\text { Upper favorable oxygen } \\
\text { concentration }\end{array}$ & $2 \%$ \\
\hline$C_{\min }$ & $\begin{array}{l}\text { Lower favorable oxygen } \\
\text { concentration }\end{array}$ & $0.3 \%$ \\
\hline$\widehat{C}_{\min }$ & $\begin{array}{l}\text { Lower detectable oxygen } \\
\text { concentration }\end{array}$ & $0.01 \%$ \\
\hline S & Length of the capillary & $5 \mathrm{~mm}$ \\
\hline
\end{tabular}

The main objective is to validate the model, by showing that it is capable of capturing experimental observations not only qualitatively but also quantitatively.

\section{Results}

This section contains a series of computer simulations of the mathematical model described in section Mathematical Model.

The model consists of advection-reaction equations for right-moving and left-moving bacteria in a capillary tube, and a diffusion-reaction equation for oxygen, which diffuses into the water from the meniscus and is consumed by bacteria. The primary computed quantities are $B(x, t)$ and $C(x, t)$, the bacteria and oxygen concentrations at location $x$ at time $t$, and the location of the left-side and right-side of the band, found as FWHM (Full Width at Half Maximum) from $B(x, t)$ at each $t$. We plot the evolution of the band in time and profiles of $B$ and $C$ at desired times, see Fig. 2a b. Some implementation details are given in subsection Numerical Implementation.

\section{Experimental validation on wild type $A$. brasilense}

We present here results of simulations of actual experiments with wild type ( $\mathrm{Sp} 7)$ A. brasilense grown in malate (as carbon source). In all the simulations, bacteria formed a stable aerotactic band. The location and width of the band agree well with those measured for microaerophilic A. brasilense cells in $[10,13]$.
Experimentally measured band location and width, for cells inoculated into the spatial gradient at the density determined by CFU counts, were respectively $407 \pm 168$ and $132 \pm 44 \mu \mathrm{m}$ at time $300 \mathrm{~s}$. Cell tracking yielded mean values for speed $v=20 \mu \mathrm{m} / \mathrm{s}$, reversal frequency before stimulation $F_{\text {min }}=0.35 / \mathrm{s}$, reversal frequency inside the band $F_{\text {max }, \text { band }}=0.96 / \mathrm{s}$, and outside the band $F_{\max }=0.65$ $/ \mathrm{s}$, at time $300 \mathrm{~s}$.

Using these values in the model, we determined the switch parameters $\widehat{C}_{\max }, C_{\max }, C_{\min }, \widehat{C}_{\min }$, which determine the forcing terms in the advection eqs. 1, 2 according to 3 and 4 (see Mathematical Model), to capture the experimentally measured band location and width. Numerical simulation of the model, with parameter values listed in Table 1, produces band left and right sides at 340.3 and $472.1 \mu \mathrm{m}$, hence band location (midpoint) 406.2 and width $131.8 \mu \mathrm{m}$. This is in remarkable agreement with the measured values of 407 and $132 \mu \mathrm{m}$.

Shown in Fig. 2a is the band evolution up to $600 \mathrm{sec}-$ onds. Figure $2 \mathrm{~b}$ shows profiles of bacterial density $B$ at times $50 \mathrm{~s}$ and $300 \mathrm{~s}$, and of oxygen concentration $C$ at $300 \mathrm{~s}$. Note that the bacterial concentration $B(x, t)$ is normalized by the initial value $B_{o}$, so $B=1$ is the initial concentration (assumed uniform in the capillary). Similarly, $C(x, t)$ is normalized by $C_{o}$, the oxygen at the meniscus. The rapid formation and stability of the band, seen in Fig. 2a, captures well the experimental observations seen in Fig. 2.

The values of the $C$-switches $\left(\widehat{C}_{\text {max }}, C_{\text {max }}, C_{\text {min }}, \widehat{C}_{\text {min }}\right)$ are effective concentrations at which switching of reversal frequencies occurs, creating a band. Their values capture the oxygen level the bacteria prefer during aerotaxis, which is difficult to measure experimentally. In the above simulation, the concentration of oxygen along the rightside of the band is $C=0.09 \%$, close to $\widehat{C}_{\text {min }}=0.01 \%$. Along the middle of the band, $C=0.88 \%$ is roughly halfway between $C_{\text {min }}=0.3 \%$ and $C_{\max }=2 \%$. And along the left-side, closer to the meniscus, $C=2.85 \%$ is just above $C_{\text {max }}$. Thus here the band forms in the oxygen range from $0.09 \%$ to $2.85 \%$, which corresponds to 1.2 to $37 \mu \mathrm{M}$ dissolved oxygen. The earlier estimate of $3-5 \mu \mathrm{M}$ [2] for preferred oxygen is very rough. It was determined first using microelectrodes that had a sensitivity limit of $1 \%$ oxygen [10]. A complimentary method used a gas proportioner to control the oxygen concentration in a gas mixture flowing into a gas chamber in which the capillary tubes were placed. The front of the band was adjacent to the meniscus when the oxygen concentration, determined by the gas proportioner, was $0.5 \%$ and the band dissipated and disappeared at oxygen concentrations lower than $0.05 \%$ in the gas mixture. While the method provided approximate numbers on oxygen concentrations, it is neither sensitive nor accurate. Furthermore, one would expect that manipulating the oxygen concentration in the 


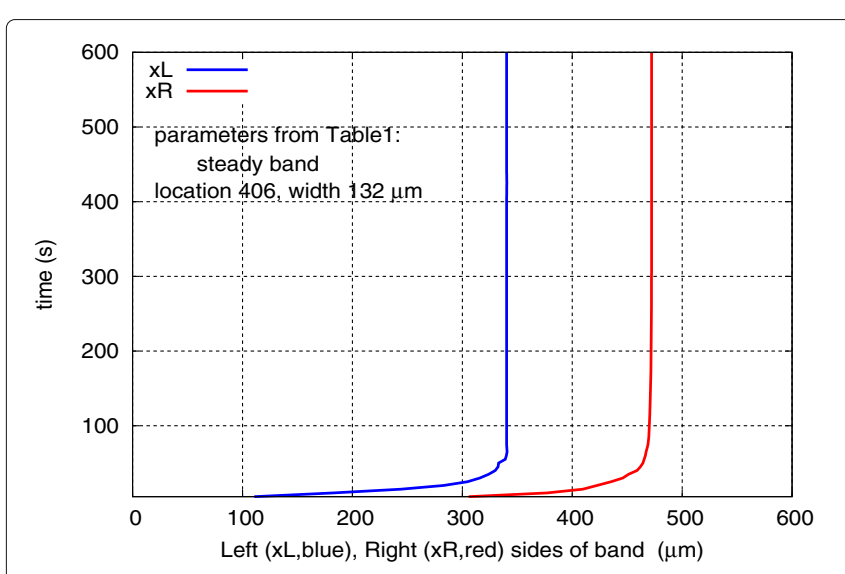

a

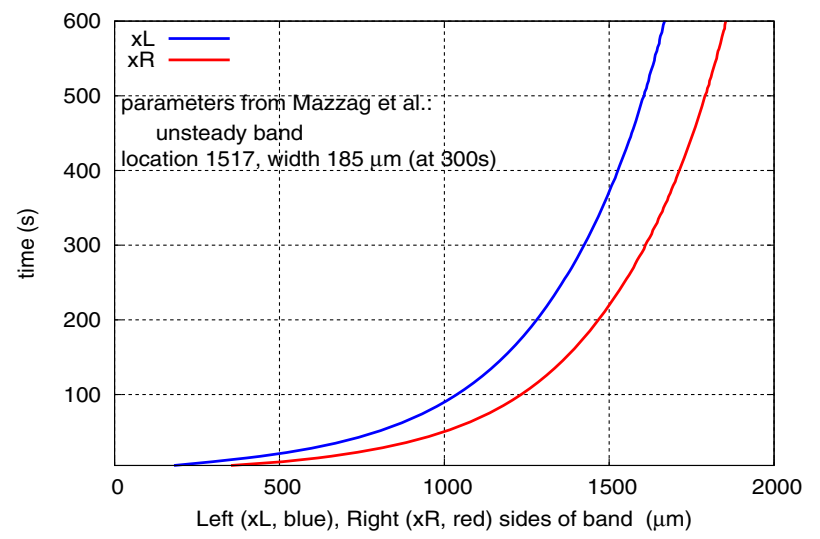

C

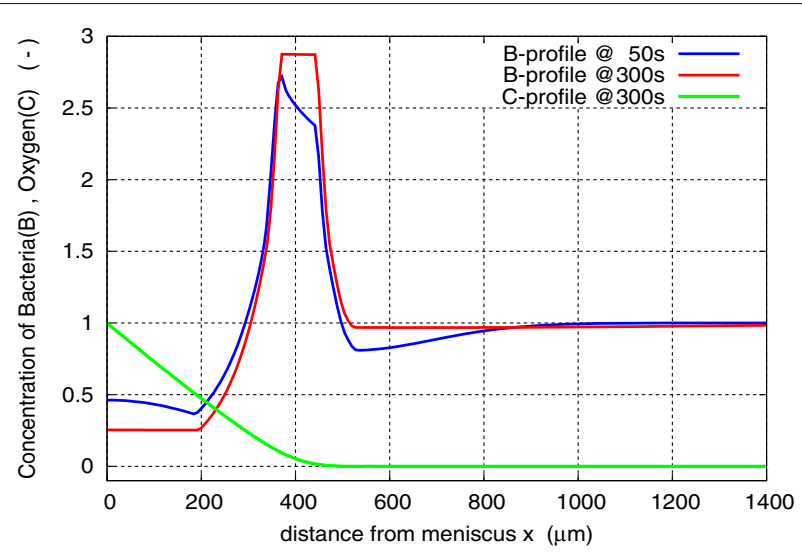

b

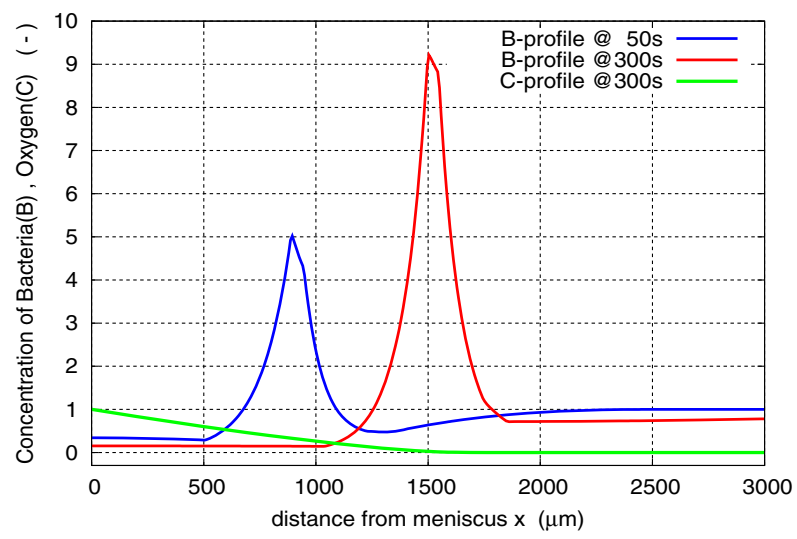

d

Fig. 2 Aerotactic band formation predicted by the model. Top row: With parameters of Table 1. The band forms and stabilizes within a minute, and remains steady, exactly as observed in experiments. Band location and width are $406 \mu \mathrm{m}$ and $132 \mu \mathrm{m}$, in excellent agreement with the experimentally measured values of 407 and $132 \mu \mathrm{m}$. Bottom row: With parameter values taken from Mazzag et al. [15]. The band is moving (not steady); location and width are 1517 and $185 \mu \mathrm{m}$ at 300 s, but 1760 and $186 \mu \mathrm{m}$ at 600 s. (a),(c): Band evolution in time: Left (blue) and Right (red) sides of the band. Note the different scales on x-axis. (b), (d): Profiles of (normalized) bacteria concentration (B) at time 50 s (blue) and 300 s (red), and of Oxygen concentration (C) at $300 \mathrm{~s}$ (green). Note the different scales on both axes

gas mixture flowing into the cell would affect the aerotaxis response and bias the outcome. Thus, the oxygen range we obtained here via the model appears reasonable.

A simulation using parameter values from Mazzag et al. [15] produces a band that keeps moving over time and does not stabilize, shown in Fig. 2c d. The parameters that differ from those in Table 1 , are: $B_{o}=1 \times$ $10^{8}$ cells $/ \mathrm{ml}, K=1 \times 10^{-9} \mu \mathrm{M} \mathrm{ml} / \mathrm{s} /$ cell, $v=40 \mu \mathrm{m} / \mathrm{s}$, $F_{\text {max }}=0.5 / \mathrm{s}, F_{\text {min }}=0.1 / \mathrm{s}, \widehat{C}_{\text {max }}=5 \%, C_{\text {max }}=0.5 \%$, and also the (Henry's Law) factor for conversion of oxygen\% in air to $\mu \mathrm{M}$ dissolved oxygen in water: $1200 \mu \mathrm{M}$ whereas we use factor 1300. Crucial parameters for getting a stable band are $K, B_{o}, C$-switches. Further replacing other parameters with ours eventually leads to Fig. $2 \mathrm{a} b$ that matches experimental measurements.

It should be noted that the model is capable of producing a great variety of band behaviors: wavy sides / smooth but moving / steady but not smooth / steady and smooth, at various locations, with various widths, all depending on combinations of parameters. No single parameter can account for any particular effect. Unwildly band behavior is not normally observed in experiments, so if they arise during simulations they are deemed unphysical, indicating poor parameters.

Parameter idendification is an "ill-posed" problem mathematically, typically uniqueness of solution and/or continuous dependence on data break down. Nevertheless, seeking $C$-switches to match both location and width of the experimentally measured band seems to constrain the system to have unique solution or no solution. In our extensive simulations we are able to find either only one combination or none at all.

\section{Band sensitivity on parameters}

Having validated the model on experimental data, we present parametric studies on the main parameters $B_{0}$, 
Table 2 Sensitivity of band location and width on parameters. Only one parameter is varied at a time, with all others at their base values as in Table 1. For ease of comparison, base value of each parameter is listed, which produces location 406, width $132 \mu \mathrm{m}$. The $\%$ changes are relative to base values. Columns 4 and 6 show sensitivity of location and width on each parameter. "Bpeak" in column 7 is the maximum bacterial concentration at $300 \mathrm{~s}$ (normalized by the initial concentration $B_{0}$ ). "Smoothness" in column 8 refers to the appearance of left- and right-side of band location vs time, as seen in Fig. 2

\begin{tabular}{|c|c|c|c|c|c|c|c|}
\hline Parameter & $\%$ change & Location $(\mu \mathrm{m})$ & $\%$ change & Width $(\mu \mathrm{m})$ & $\%$ change & Bpeak & Smoothness \\
\hline$B_{0}=1 \times 10^{9} \mathrm{cells} / \mathrm{ml}$ & $+50 \%$ & 352 & $-13 \%$ & 114 & $-14 \%$ & 2.6 & Smooth \\
\hline$B_{0}=7 \times 10^{8} \mathrm{cells} / \mathrm{ml}$ & base value & 406 & - & 132 & - & 2.9 & Smooth \\
\hline$B_{0}=3 \times 10^{8} \mathrm{cells} / \mathrm{ml}$ & $-50 \%$ & 582 & $+43 \%$ & 156 & $+18 \%$ & 3.9 & Smooth \\
\hline$C_{0}=30 \%$ & $+50 \%$ & 527 & $+30 \%$ & 122 & $-8 \%$ & 3.2 & $\sim$ smooth \\
\hline$C_{0}=21 \%$ & base value & 406 & - & 132 & - & 2.9 & Smooth \\
\hline$C_{0}=10 \%$ & $-50 \%$ & 227 & $-44 \%$ & 133 & $1 \%$ & 2.6 & smooth $>220$ s \\
\hline$D=2.5 \times 10^{3} \mu \mathrm{m}^{2} / \mathrm{s}$ & $+25 \%$ & 449 & $+11 \%$ & 140 & $+6 \%$ & 3.1 & Smooth \\
\hline$D=2.0 \times 10^{3} \mu \mathrm{m}^{2} / \mathrm{s}$ & base value & 406 & - & 132 & - & 2.9 & Smooth \\
\hline$D=1.5 \times 10^{3} \mu \mathrm{m}^{2} / \mathrm{s}$ & $-25 \%$ & 359 & $-12 \%$ & 114 & $-13 \%$ & 2.7 & Smooth $>200$ s \\
\hline $\mathrm{K}=6 \times 10^{-9} \mu \mathrm{M} \mathrm{ml} / \mathrm{s} / \mathrm{cell}$ & $+50 \%$ & 344 & $-15 \%$ & 114 & $-14 \%$ & 2.6 & Smooth $>100 \mathrm{~s}$ \\
\hline$K=4 \times 10^{-9} \mu \mathrm{M} \mathrm{ml} / \mathrm{s} / \mathrm{cell}$ & base value & 406 & - & 132 & - & 2.9 & Smooth \\
\hline $\mathrm{K}=2 \times 10^{-9} \mu \mathrm{M} \mathrm{ml} / \mathrm{s} /$ cell & $-50 \%$ & 547 & $+32 \%$ & 149 & $+13 \%$ & 3.7 & Smooth $>110$ s \\
\hline$v=30 \mu \mathrm{m} / \mathrm{s}$ & $+50 \%$ & 429 & $+6 \%$ & 144 & $+9 \%$ & 2.4 & $\sim$ smooth \\
\hline$v=20 \mu \mathrm{m} / \mathrm{s}$ & Base value & 406 & - & 132 & - & 2.9 & Smooth \\
\hline$v=10 \mu \mathrm{m} / \mathrm{s}$ & $-50 \%$ & 382 & $-6 \%$ & 98 & $-26 \%$ & 4.2 & Smooth $>200 \mathrm{~s}$ \\
\hline$F_{\max }=0.85 / \mathrm{s}$ & $+30 \%$ & 387 & $-5 \%$ & 125 & $-5 \%$ & 3.5 & Smooth \\
\hline$F_{\max }=0.65 / \mathrm{s}$ & Base value & 406 & - & 132 & - & 2.9 & Smooth \\
\hline$F_{\max }=0.45 / \mathrm{s}$ & $-30 \%$ & 445 & $+10 \%$ & 133 & $0 \%$ & 1.9 & $\sim$ smooth \\
\hline$F_{\min }=0.45 / \mathrm{s}$ & $+30 \%$ & 426 & $+5 \%$ & 141 & $+7 \%$ & 2.4 & Smooth \\
\hline$F_{\min }=0.35 / \mathrm{s}$ & Base value & 406 & - & 132 & - & 2.9 & Smooth \\
\hline$F_{\min }=0.25 / \mathrm{s}$ & $-30 \%$ & 387 & $-5 \%$ & 117 & $-11 \%$ & 3.5 & Smooth \\
\hline$C_{\max }=3 \%$ & $+50 \%$ & 383 & $-6 \%$ & 159 & $+21 \%$ & 2.6 & 〜smooth \\
\hline$C_{\max }=2 \%$ & Base value & 406 & - & 132 & - & 2.9 & Smooth \\
\hline$C_{\max }=1 \%$ & $-50 \%$ & 457 & $+13 \%$ & 94 & $-29 \%$ & 3.4 & $\sim$ smooth \\
\hline$C_{\min }=0.45 \%$ & $+50 \%$ & 391 & $-4 \%$ & 115 & $-13 \%$ & 3.2 & Smooth \\
\hline$C_{\min }=0.3 \%$ & Base value & 406 & - & 132 & - & 2.9 & Smooth \\
\hline$C_{\min }=0.15 \%$ & $-50 \%$ & 437 & $+8 \%$ & 148 & $+12 \%$ & 2.5 & $\sim$ smooth \\
\hline$\widehat{C}_{\max }=15 \%$ & $+50 \%$ & 402 & $-1 \%$ & 126 & $-5 \%$ & 3.2 & Smooth $>120 \mathrm{~s}$ \\
\hline$\widehat{C}_{\max }=10 \%$ & Base value & 406 & - & 132 & - & 2.9 & Smooth \\
\hline$\widehat{C}_{\max }=5 \%$ & $-50 \%$ & 418 & $+3 \%$ & 125 & $-6 \%$ & 2.4 & 〜smooth \\
\hline$\widehat{C}_{\min }=0.015 \%$ & $+50 \%$ & 406 & $0 \%$ & 132 & $0 \%$ & 2.9 & Smooth \\
\hline$\widehat{C}_{\min }=0.010 \%$ & Base value & 406 & - & 132 & - & 2.9 & Smooth \\
\hline$\widehat{C}_{\min }=0.005 \%$ & $-50 \%$ & 406 & $0 \%$ & 132 & $0 \%$ & 2.9 & Smooth \\
\hline
\end{tabular}

$C_{o}, K, v, F_{\text {max }}, F_{\text {min }}$, and on the $C$-switches: $\widehat{C}_{\text {max }}, C_{\text {max }}$, $C_{\text {min }}, \widehat{C}_{\text {min }}$, to see how increasing or decreasing each one affects the band location and width, and by how much. The results are listed in Table 2 .

For ease of comparison, the base value (from Table 1) of each parameter is listed, that produces location 406, width $132 \mu \mathrm{m}$. Only one parameter is varied at a time, with all others at their base values. The \% changes are relative to base values. It should be noted that the sensitivities shown in Table 2 are local about the base values. They may be different about some other base state.

In the following subsections we discuss some of the rows in Table 2 to point out the meaning of the entries. Similar considerations apply to the rest of the parameters in Table 2. 


\section{Effect of bacterial density $B_{o}$}

Band location and width depend strongly on the total bacteria density. Inreasing $B_{o}$ by $50 \%$ of base value to $1 \times 10^{9}$ cells $/ \mathrm{ml}$, the band formed closer to the meniscus, at $352 \mu \mathrm{m}(-13 \%$ change), with narrower width (114 $\mu \mathrm{m},-14 \%$ change). The maximum of the bacterial distribution in the band (Bpeak, in column 7), is now 2.6, meaning $2.6 \times B_{o}$, a bit lower than the 2.9 peak of the base case. The entry "smooth" in column 8 refers to the shape of band sides in a plot like Fig. 2a. Changing $B_{o}$ by $-50 \%$ to $B_{o}=3 \times 10^{8}$ cells $/ \mathrm{ml}$, the band formed much further (at $582 \mu \mathrm{m},+43 \%$ change), with wider width (156 $\mu \mathrm{m},+18 \%$ change). Thus, band position and width are both decreasing functions of $B_{o}$, with location being affected more strongly than width, especially at lower $B_{o}$. These are in agreement with general experimental observations.

\section{Effect of Oxygen at the capillary opening, $C_{o}$}

Band location depends strongly on the oxygen concentration at the meniscus opening, which affects the oxygen gradient into the capillary. When $C_{o}$ was increased to $30 \%$, the band formed much further, at $527 \mu \mathrm{m}$ ( $+30 \%$ change), but with narrower width $122 \mu \mathrm{m}$ ( $-8 \%$ change). The entry " smooth" in column 8 means the band sides are mostly smooth but with a few step-like movements. When $C_{o}$ was halved to $10 \%$, the band formed much closer to the meniscus, at $227 \mu \mathrm{m}$ (-44\% change), with unchanged width. The entry "smooth $>220 \mathrm{~s}$ " means the band sides show some step-like movements early on and become smooth after time 220s. Thus, band position is an increasing function of $C_{o}$, but band width is little affected by $C_{o}$. The effect of increasing/decreasing $C_{o}$ on band location is as one would expect: increasing $C_{o}$ raises the oxygen concentration profile $C(x)$, so the switch values $C_{\max }, C_{\min }$ and the band occur further to the right.

\section{Effect of consumption rate $K$}

Band location and width depend considerably on oxygen consumption rate, as is to be expected. When $K$ was increased by $50 \%$ to $K=6 \times 10^{-9} \mu \mathrm{M} \mathrm{ml} / \mathrm{s}$ cell, the band formed closer to the meniscus at $344 \mu \mathrm{m}(-15 \%$ change $)$, with narrower width $114 \mu \mathrm{m}(-14 \%$ change). When $K$ was decreased $50 \%$ to $K=2 \times 10^{-9} \mu \mathrm{Mml} / \mathrm{s}$ cell, the band formed much further away, at $547 \mu \mathrm{m}$, a considerable change of $+32 \%$. It had wider width of $149 \mu \mathrm{m}(+13 \%$ change). Thus, band position and width are decreasing functions of oxygen consumption rate $K$, with location being affected much more than the width. Again, the predicted behavior aligns with experimental observations.

\section{Discussion}

The ability to navigate gradients of oxygen is key to regulate metabolic activities of bacteria with a range of lifestyles. It is thus not surprising to observe that aerotaxis is a widespread behavior in bacteria and Archaea [2].

Several mathematical models have been developed to recapitulate the movement of bacteria in oxygen gradients. The models developed for bacteria that track higher concentrations of oxygen such as B. subtilis [16] or which prefer lower oxygen concentrations such as Desulfovibrio desulfuricans [17] are not appropriate for A. brasilense because the aerotaxis strategy of these organisms is distinct. B. subtilis detects oxygen directly and navigates toward elevated oxygen concentrations while $D$. desulfuricans is a strict anaerobe that forms a band at the oxic-anoxic interface with the band being far less stable than that observed for A. brasilense.

When we attempted to use the previously developed model for $A$. brasilense aerotaxis band formation by Mazzag et al. [15], we could not produce a stable aerotactic band, despite this feature being characteristic of the $A$. brasilense aerotaxis response [10,11]. The model and experimental values used here provide a robust model that captures all significant features of $A$. brasilense aerotaxis band formation.

Our model predicts that cell density $\left(B_{o}\right)$, oxygen concentration at the meniscus $\left(C_{o}\right)$, and oxygen consumption rate $(K)$ have significant effect on the location of the aerotactic band, but speed does not. On the other hand, width of the band is most sensitive to cell density $\left(B_{o}\right)$ and speed $(v)$, but not to $C_{o}$. In addition to experimental data validating at least some of these observations here, Barak et al. [18] demonstrated that increasing the oxygen concentration available at the capillary opening delayed band formation, and led to an increase in the number of attracted bacteria to the band, i.e., the band became thicker over time.

The tight aerotactic band formed by $A$. brasilense in gradients of oxygen depends on the abilty to sense oxygen as both an attractant and a repellent. A. brasilense senses very low or very high oxygen concentrations as repellents and motile cells navigate the gradients to stay away from these two strong repellents to locate themselves where oxygen is an attractant [10]. These opposing behaviors are captured in the model described here and by our experimental data indicating a very high probability of reversal in swimming direction for cells within the band.

Determining the $C$-switch values computationally, captures the narrow range of oxygen concentrations the bacteria prefer to congregate in, forming an aerotactic band. As this is difficult to do reliably in experiments, it is a major advantage of the model and approach described here. In the validated model the band forms between 1.2 and $37 \mu \mathrm{M}$ dissolved oxygen. Given our findings and observed effects of respiration rates and density, we expect this range to vary with experimental conditions. 
One would expect fluid mixing induced by swimming cells to increase the diffusion coefficient of oxygen in water; the issue is how significant it would be. In a rather thorough paper on the subject, Kasyap et al. [19] estimated the hydrodynamic diffusivity induced by swimming bacteria, and conclude that "bacteria induced mixing is irrelevant for small molecules". Indeed, their formula for hydrodynamic diffusivity (for oxygen, using our parameter values) yields $0.7 \mathrm{\mu m}^{2} / \mathrm{s}$ inside the band and $0.9 \mu \mathrm{m}^{2} / \mathrm{s}$ outside the band. These are indeed negligible compared to the molecular diffusivity $D=2000 \mu \mathrm{m}^{2} / \mathrm{s}$ of oxygen in water.

Motivated by this question, in Table 2 we examine the effect of a large $\pm 25 \%$ change in $D$ to gauge uncertainty. It turns out that a large $+25 \%$ increase of $D$ would result in a rather modest $+10 \%$ increase in band location, and would have no effect on width. (The case of $-25 \%$ change is included for completeness, it is not expected to arise).

\section{Conclusions}

A mathematical model for aerotaxis band formation was presented and validated on experimental data for Azospirillum brasilense. A spatial gradient assay for aerotaxis and cell tracking provide values for swimming speed and reversal frequencies, which are parameters in the model. Four other model parameters (that cannot be measured experimentally) were determined computationally so as to match measured band location and width. With these parameters, the model captures all significant features of $A$. brasilense aerotaxis band formation. The simulation reveals that wild-type $\mathrm{Sp} 7$ A. brasilense forms the band in the range of 1.2 to $37 \mu \mathrm{M}$ dissolved oxygen.

Parametric studies predict that band location depends strongly on cell density $\left(B_{o}\right)$, oxygen concentration at the meniscus $\left(C_{o}\right)$, and oxygen consumption rate $(K)$, but not on swimming speed $(v)$. On the other hand, width of the band is most sensitive to $B_{o}, v$, and $K$, but not to $C_{o}$.

\section{Methods}

\section{Strain growth conditions}

The motile soil alphaproteobacterium A. brasilense strain Sp7 ([20]) was used in these experiments. A. brasilense cells were routinely grown in liquid MMAB (Minimal Medium for Azzospirillum brasilense [21]), supplemented with malate $(10 \mathrm{mM})$ and ammonium chloride $(18.7 \mathrm{mM})$, as previously described [14]. For Colony Forming Units (CFU) counts, liquid cultures were grown to the desired optical density at 600 $\mathrm{nm}\left(\mathrm{OD}_{600}\right)$. One $\mathrm{ml}$ aliquots were taken and serially diluted 10-fold and plated on TY medium (Tryptone $10 \mathrm{~g} / \mathrm{l}$, Yeast Extract $5 \mathrm{~g} / \mathrm{l}$ ) with ampicillin $(200 \mu \mathrm{g} / \mathrm{ml})$.

\section{Spatial gradient assay for aerotaxis}

Cells were grown to an $\mathrm{OD}_{600}$ of 0.4 - 0.6 (exponential phase of growth) in MMAB supplemented with malate and ammonium. Cultures were washed 3 times with chemotaxis buffer and standardized to an $\mathrm{OD}_{600}$ of 0.5 [22]. One $\mathrm{ml}$ of this culture of motile cells were gently washed with sterile chemotaxis buffer by centrifugation (5000 rpm for 3 minutes) and resuspended in $100 \mu \mathrm{l}$ chemotaxis buffer containing malate. Over 95\% of cells remained motile under these conditions. Cells were transferred to an optically flat microcapillary tube (inner dimensions $0.1 \times 2 \times 50 \mathrm{~mm}$, Vitro Dynamics, Inc., Rockaway, NJ) by immersing a capillary tube into the suspension of motile cells. The cells were equilibrated in a gas perfusion chamber with $\mathrm{N}_{2}$ gas for 3 minutes, then air (21\% oxygen) was introduced, and aerotactic band formation was visualized under a light microscope at $4 \times$ magnification, and videotaped at $30 \mathrm{fps}$. Upon formation of a stable band [13], band location was measured at midheight of the capillary from (surface of) the meniscus to center of the band; band width was also measured at the same mid-height. Time to stable band formation was also measured.

Snapshots of band formation in such a spatial gradient assay are shown in Fig. 1. The band forms very fast and stabilizes within a couple of minutes.

\section{Single cell tracking}

To determine the swimming reversal frequency and swimming speed of cells within and outside the aerotactic band, a digital recording (at $40 \times$ magnification) of the aerotactic band formed by wild type strains under the conditions described above was analyzed using CellTrak (Santa Rosa, CA), following the procedure described in [11]. A minimum of 100 individual tracks were analyzed and the average values as well as minimum and maximal values were determined from these data sets and used for mathematical modeling.

\section{Mathematical Model}

Various modeling approaches for chemotaxis exist: Ordinary Differential Equation (ODE) models for signaling pathways [23-25]; Partial Differential Equation (PDE) models of various types for chemotactic movement, most commonly Keller-Segel type models [26]; stochastic models of various types [27-29]; and agent-based models [30, 31].

The most extensively studied mathematical models for chemotaxis are Keller-Segel type models, named after the 1971 work of Evelyn Keller and Lee Segel [26], even though similar models were derived already by C.S. Patlak in 1953 [32]. Such models describe evolution of bacterial density by a parabolic PDE involving an antidiffusion "chemotaxis term" proportional to the gradient 
of the chemoattractant, thus allowing movement up-thegradient, the most prominent feature of chemotaxis. It has been shown that in 2 and higher (space) dimensions, under certain conditions, finite-time blow-up may occur which is clearly unphysical (sometimes interpreted as "overcrowding") [33]. An excellent summary of mathematical results on Keller-Segel models up to 2004 can be found in $[34,35]$.

The type of model employed here was initially formulated for chemotaxis by Lee Segel [36, 37], and it is more physical (and more "primitive", in the sense that under appropriate assumptions it reduces to the Keller-Segel model). It was adapted for aerotaxis by Mazzag et al. [15] to model energy taxis $[10,11,38]$. A great advantage of the model is that it incorporates experimentally measurable parameters, namely swimming speed and reversal frequencies, as it will be described below. While [15] captured some of the features of the aerotaxis response of A. brasilense, it failed to produce a stable (not moving) aerotactic band, which is typical of that formed by $A$. brasilense.

Below, we present in full detail the basic mathematical model, which is a somewhat simplified version of [15], and then we mention some features of our numerical implementation.

\section{Swimming of the Bacteria}

We formulate a system of partial differential equations that describe movement of bacteria whose reversal frequency is regulated by local oxygen concentration. We consider one-dimensional movement (along the $\mathrm{x}$-axis) in an interval $0 \leq x \leq S$. The advection terms describe the directed swimming of bacteria with speed $v$, while the reaction terms denote the turning of bacteria at frequencies $f_{R L}$ and $f_{L R} . R(x, t)$ and $L(x, t)$ are the number (densities) of right-moving and left-moving bacteria at position $x$ and time $t$, respectively.

$$
\begin{aligned}
& \frac{\partial R(x, t)}{\partial t}+v \frac{\partial R(x, t)}{\partial x}=-f_{R L} R(x, t)+f_{L R} L(x, t), \\
& \frac{\partial L(x, t)}{\partial t}-v \frac{\partial L(x, t)}{\partial x}=+f_{R L} R(x, t)-f_{L R} L(x, t),
\end{aligned}
$$

where $v$ is the (constant) swimming speed, $f_{R L}$ and $f_{L R}$ are the probabilities with which bacteria reverse their direction from to Right to Left and from Left to Right, respectively, given by

$$
\begin{aligned}
& f_{R L}=\left\{\begin{array}{lll}
F_{\text {max }} \text { if } & \widehat{C}_{\text {min }}<C<C_{\text {max }}, \\
F_{\text {min }} \text { if } & C<\widehat{C}_{\text {min }} \text { or } C>C_{\text {max }},
\end{array}\right. \\
& f_{L R}=\left\{\begin{array}{lll}
F_{\text {max }} \text { if } & C_{\text {min }}<C<\widehat{C}_{\text {max }}, \\
F_{\text {min }} \text { if } & C<C_{\text {min }} \text { or } C>\widehat{C}_{\text {max }} .
\end{array}\right.
\end{aligned}
$$

Here $F_{\text {max }}$ and $F_{\text {min }}$ are maximum and minimum reversal frequencies, respectively, and $\widehat{C}_{\text {min }}<C_{\text {min }}<C_{\text {max }}<$ $\widehat{C}_{\text {max }}$ are specified switch values of oxygen concentration $C$ at which frequencies change from low $F_{\min }$ to high $F_{\text {max }}$ and vice versa. The formulae are depicted in Fig. 3.

In our implementation, we actually use different values for $F_{\text {max }}$ inside and outside the band, which are found experimentally, see Table 1 . The concentration of bacteria, $B(x, t)$, is the total number of right- and left-moving cells:

$$
B(x, t)=R(x, t)+L(x, t) .
$$

Cell reproduction is much slower than band formation time-scale and it is ignored. Initially, $R(x, 0)=R_{o}(x)$

\section{reversal}

frequency

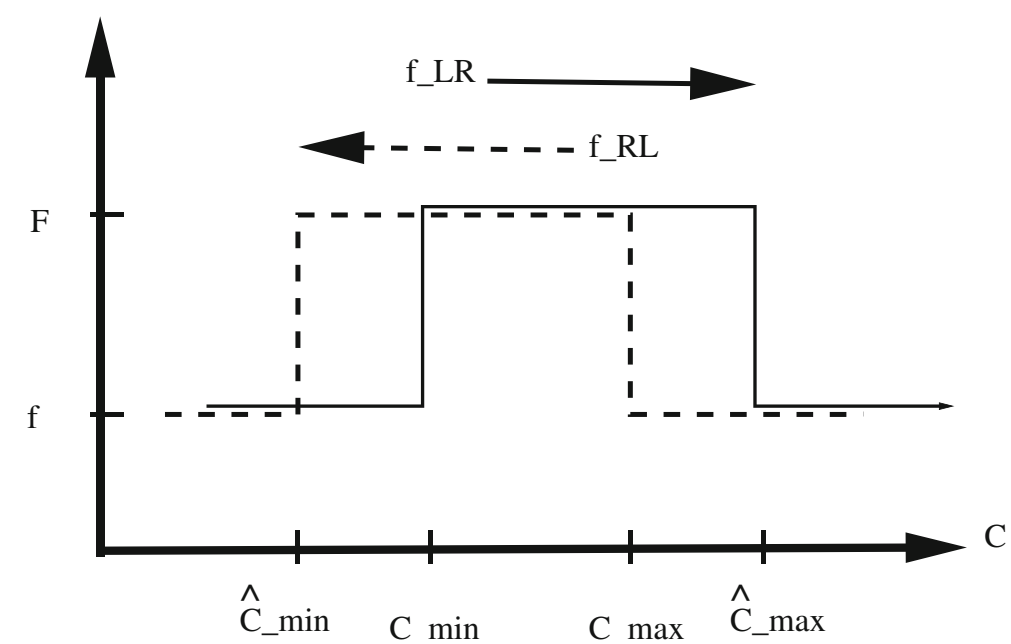

Fig. 3 Reversal frequency of right swimming (solid line) and left swimming (dashed line) cells, depicting formulas (3) and (4), for setting $f_{R L}$ and $f_{L R}$ in the model 
and $L(x, 0)=L_{o}(x)$ in $[0, S]$, for some initial distributions $R_{o}(x)$ and $L_{o}(x)$. At the left boundary all left-moving cells turn to the right, and at the right boundary all rightmoving cells turn to the left: $R(0, t)=L(0, t)$ and $R(S, t)=L(S, t)$. These boundary conditions ensure that there is no depletion of bacteria, thus the total number of bacteria in the capillary $[0, S]$ remains constant and equal to the initial number

$$
\int_{0}^{S} B(x, t) d x=\text { const. }=B_{o}=R_{o}+L_{o} .
$$

\section{Diffusion of Oxygen}

The oxygen concentration $C(x, t)$ in the capillary $[0, S]$ is determined by a diffusion-reaction equation that accounts for the consumption of oxygen by the bacteria:

$$
\frac{\partial C(x, t)}{\partial t}=D \frac{\partial^{2} C(x, t)}{\partial x^{2}}-K \theta(C(x, t)) B(x, t),
$$

where $B(x, t)$ is the concentration of bacteria (Eq. 5 ), $K$ is the rate of consumption of oxygen by bacteria, and $D$ is diffusion coefficient of oxygen in water. To ensure there is no consumption after oxygen depletion, $\theta(C)$ is set as

$$
\theta(C(x, t))=\left\{\begin{array}{l}
1 \text { if } C(x, t)>0 \\
0 \text { if } C(x, t) \leq 0
\end{array}\right.
$$

Initially there is no oxygen in the capillary, so the initial condition is

$$
C(x, 0)=0 \text { for all } 0 \leq x \leq S .
$$

At the open end $x=0$ the oxygen concentration is a specified value $C_{o}$, while the other end of the capillary is sealed (with wax) to prevent oxygen from entering or leaving. Thus the boundary conditions at $x=0$ and $x=S$ are

$$
C(0, t)=C_{o}, \quad \frac{\partial C(x, t)}{\partial x}=0 \quad \text { at } x=S .
$$

\section{Numerical Implementation}

The system of partial differential eqs. (1), (2), (7) was undimensionalized and solved numerically in Fortran 90. The advection equations were discretized with an upwind Finite Volume scheme and forward Euler time discretization. The diffusion equation was also discretized by Finite Volumes with forward Euler time discretization.

In the simulations, we used capillary length $S=5 \mathrm{~mm}$, which is already far away from where the band forms and does not affect the numerical results. The capillary, occupying the interval $[0, S]$ was discretized into 640 control volumes (128 per $\mathrm{mm}$ ), and the time-step was chosen judiciously and adaptively to ensure numerical stability and non-negative concentrations.

We note here some features in our implementation and differences with Mazzag et al. [15].

(1) In conformance with measurements, reversal frequency is higher inside the band instead of lower. Thus
Fig. 3 is a "flipped" version of Fig. 2c in [15].

(2) Reversal frequencies are applied separately inside the band and outside the band (different values for $F_{\text {max }}$ may be used inside and outside the band). This was motivated by experimental measurements, and necessitated calculation of band location at each time-step in the numerical implementation. The band is calculated from the bacterial distribution $B\left(x, t_{n}\right)$ at each time-step $t_{n}$, as FWHM (Full Width at Half Maximum), a common practice in many fields. This is done by intersecting the density profile by a horizontal line at half-maximum to determine the left side $(x L)$ and right side $(x R)$ of the band at time $t_{n}$, which are plotted in Fig. 2a.

(3) In the simulations we use much finer space grid $(128 / \mathrm{mm}$ instead of $40 / \mathrm{mm}$ used in [15]) which noticeably affects the calculated band location.

\section{Abbreviations}

CCW: Counter clock wise rotation of flagellar motor; CFU: Colony forming units; CW: Clock wise rotation of flagellar motor; MMAB: Minimal medium for azospirillum brasilense; ODE: Ordinary differential equation; $\mathrm{OD}_{600}$ : Optical density at $600 \mathrm{~nm}$; PDE: Partial differential equation

\section{Acknowledgements}

Not applicable.

\section{Funding}

This work was supported by NSF-MCB 1330344 grant to GA and VA. LON was supported by NIH R25GM086761. Any opinions, findings, conclusions, or recommendations expressed in this material are those of the authors and do not necessarily reflect the views of the National Science Foundation or $\mathrm{NIH}$.

\section{Availability of data and materials}

The datasets and computer codes used in the current study are available from the corresponding author on reasonable request.

\section{Authors' contributions}

ME, VA, GA conceived the study, and wrote the paper. LON, GA planned the experiments, which were carried out by LON. ME, VA developed the mathematical model and carried out the numerical simulations. All authors have read and approved the manuscript.

\section{Ethics approval and consent to participate}

Not applicable.

Consent for publication

Not applicable.

\section{Competing interests}

The authors declare that they have no competing interests.

\section{Publisher's Note}

Springer Nature remains neutral with regard to jurisdictional claims in published maps and institutional affiliations.

\section{Author details \\ ${ }^{1}$ Mathematics, University of Tennessee, 1403 Circle Dr, Knoxville, TN, 37996 USA. ${ }^{2}$ Biochemistry and Cellular \& Molecular Biology, University of Tennessee,} 1311 Cumberland Ave, Knoxville, TN, 37996 USA.

Received: 3 October 2018 Accepted: 30 April 2019

Published online: 17 May 2019

References

1. Bi S, Sourjik V. Stimulus sensing and signal processing in bacterial chemotaxis. Curr Opin Microbiol. 2018;45:22-9. https://doi.org/10.1016/j. mib.2018.02.002 
2. Taylor B, Zhulin I, Johnson M. Aerotaxis and other energy-sensing behavior in bacteria. Annual Rev Microbiol. 1999;53:103-28. https://doi. org/10.1146/annurev.micro.53.1.103.

3. Scharf $B$, Hynes M, Alexandre G. Chemotaxis signaling systems in model beneficial plant-bacteria associations. Plant Mol Biol. 2016;90(6):549-59. https://doi.org/10.1007/s11103-016-0432-4.

4. Engelmann T. Neue methode zur untersuchung der sauerstoffausscheidung pflanzlicher und tierischer organismen. Pflugers Arch Gesammte Physiol Menschen Tiere. 1881;25:285-92.

5. Engelmann T. Zur biologie der schizomyceten. Pflugers Arch Gesammte Physiol Menschen Tiere. 1881;26:537-45.

6. Beijerinck M. Ueber atmungsfiguren beweglicher bakterien. Zentrabl Bakteriol Parasitenkd. 1893;14:827-45.

7. Hou S, Larsen R, Boudko D, Riley C, Karatan E, Zimmer M, Ordal G, Alam M. Myoglobin-like aerotaxis transducers in Archaea and Bacteria. Nature. 2000;403:540-4. https://doi.org/10.1038/35000570.

8. Bibikov S, Biran R, Rudd K, Parkinson J. A signal transducer for aerotaxis in Escherichia coli. J Bacteriol. 1997;179(12):4075-9. https://doi.org/10. 1128/jb.179.12.4075-4079.1997.

9. Rebbapragada A, Johnson M, Harding G, Zuccharelli A, Fletcher H, Zhulin I, Taylor B. The aer protein and the serine chemoreceptor Tsr independently sense intracellular energy levels and transduce oxygen, redox, and energy signals for Escherichia coli behavior. PNAS. 1997;94(20): 10541-6. https://doi.org/10.1073/pnas.94.20.10541.

10. Zhulin I, Bespalov V, Johnson M, Taylor B. Oxygen taxis and proton motive force in Azospirillum brasilense. J Bacteriol. 1996;178(17): 5199-204. https://doi.org/10.1128/jb.178.17.5199-5204.1996.

11. Alexandre G, Greer S, Zhulin I. Energy taxis is the dominant behavior in Azospirillum brasilense. J Bacteriol. 2000;182(21):6042-8. https://doi.org/ 10.1128/JB.182.21.6042-6048.2000.

12. Xie Z, Ulrich L, Zhulin I, Alexandre G. Pas domain containing chemoreceptor couples dynamic changes in metabolism with chemotaxis. PNAS. 2010;107:2235-40. https://doi.org/10.1073/pnas. 0910055107.

13. O'Neal L, Ryu M, Gomelsky M, Alexandre G. Optogenetic manipulation of cyclic di-GMP (c-di-GMP) levels reveals the role of c-di-GMP in regulating aerotaxis receptor activity in Azosprillum brasilense. (c-di-GMP) J Bacteriol. 2017;199(18):00020-17. https://doi.org/10.1128/JB.00020-17.

14. Bible A, Stephens B, Ortega D, Xie Z, Alexandre G. Function of a chemotaxis-like signal transduction pathway in modulating motility, cell clumping, and cell length in the alphaproteobacterium Azospirillum brasilense. J Bacteriol. 2008;190:6365-75. https://doi.org/10.1128/JB. 00734-08.

15. Mazzag B, Zhulin I, Mogilner A. Model of bacterial band formation in aerotaxis. Biophysical J. 2003;85(6):3558-74. https://doi.org/10.1016/ S0006-3495(03)74775-4.

16. Menolascina F, Stocker R, Sontag E. In-vivo indentification and control of aerotaxis in Bacillus subtilis. In: 2016 IEEE 55th Conference on Decision and Control (CDC). Las Vegas: IEEE; 2016. p. 764-9. https://doi.org/10. 1109/CDC.2016.7798360.

17. Fischer J, Cypionka H. Analysis of aerotactic band formation by Desulfovibrio desulfuricans in a stopped-flow diffusion chamber. FEMS Microbiol Ecol. 2006;55(2):186-94. https://doi.org/10.1111/j.1574-695X. 2005.00024.x

18. Barak R, Nur I, Okon Y. Detection of chemotaxis in Azospirillum brasilense. J Appl Bacteriol. 1983;53:399-403. https://doi.org/10.1111/j. 1365-2672.1983.tb02634.x.

19. Kasyap T, Koch D, Wu M. Hydrodynamic tracer diffusion in suspensions of swimming bacteria. Phys Fluids. 2014;26:081901. https://doi.org/10. 1063/1.4891570.

20. Tarrand J, Krieg N, Dobereiner J. A taxonomic study of the Spirillum lipoferum group, with descriptions of a new genus, Azospirillum gen nov and two species, Azospirillum lipoferum (beijerinck) comb nov and Azospirillum brasilense sp nov. Can J Microbiol. 1978;24:967-80.

21. Vanstockem M, Michiels K, Vanderleyden J, Gool AV. Transposon mutagenesis of Azospirillum brasilense and Azospirillum lipoferum: Physical analysis of Tn5 and Tn5-mob insertion mutants. Appl Environ Microbiol. 1987;53(2):410-5.

22. Gullett JM, O'Neal L, Mukerhjee T, Alexandre G. Azospirillum brasilense: Laboratory maintenance and genetic manipulation. Curr Protoc Microbiol. 2017;47:3E.2.1-3E.2.17. https://doi.org/10.1002/cpmc.39.
23. Mello B, Tu Y. Perfect and near-perfect adaptation in a model of bacterial chemotaxis. Biophysics J. 2003;84(5):2943-56. https://doi.org/10.1016/ S0006-3495(03)70021-6.

24. Frankel N, Pontius W, Dufour $Y$, Long J, Hernandez-Nunez L, Emonet $T$. Adaptability of non-genetic diversity in bacterial chemotaxis. eLIFE. 2014;3:03526. https://doi.org/10.7554/eLife.03526.

25. Elmas M, Mukherjee T, Alexiades V, Alexandre G. A two pathways model for chemotactic signaling in Azospirillum brasilense. Neural, Parallel, Sci Comput. 2017;25:345-57.

26. Keller E, Segel L. Model for chemotaxis. J Theor Biol. 1971;30:225-34.

27. Koumoutsakos P, Bayati B, Milde F, Tauriello G. Particle simulations of morphogenesis. Math Models Methods Appl Sci. 2011;21:955-1006. https://doi.org/10.1142/S021820251100543X.

28. Taktikos J, Zaburdaev V, Stark H. Collective dynamics of model microorganisms with chemotactic signaling. Phys Rev E. 2012;85:051901. https://doi.org/10.1103/PhysRevE.85.051901.

29. Curk T, Marenduzzo D, Dobnikar J. Chemotactic sensing towards ambient and secreted attractant drives collective behavior of E. coli. PLoS ONE. 2013;8:74878. https://doi.org/10.1371/journal.pone.0074878.

30. Kaul H, Cui Z, Ventikos Y. A multi-paradigm modeling framework to simulate dynamic reciprocity in a bioreactor. PLOS ONE. 2013;8:59671. https://doi.org/10.1371/journal.pone.0059671.

31. Bogle G, Dunbar P. On-latice simulation of t cell motility, chemotaxis, and trafficking in the lymph node paracortex. PLOS ONE. 2012;7:45258. https://doi.org/10.1371/journal.pone.0045258.

32. Patlak C. Random walk with persistence and external bias. Bull Math Biophys. 1953;15:311-38. https://doi.org/10.1007/BF02476407.

33. Fatkullin I. A study of blow-ups in the Keller-Segel model of chemotaxis. Nonlinearity. 2013;26:81-94. https://doi.org/10.1088/0951-7715/26/1/81.

34. Horstmann D. The Keller-Segel model in chemotaxis and its consequences i. Jahresbericht der DMV. 2003;105:103-65.

35. Horstmann D. The Keller-Segel model in chemotaxis and its consequences ii. Jahresbericht der DMV. 2004;106:51-69.

36. Segel L. Incorporation of receptor kinetics into a model for bacterial chemotaxis. J Theor Biol. 1976;57(1):23-42. https://doi.org/10.1016/ S0022-5193(76)80004-5.

37. Segel L. A theoretical study of receptor mechanisms in bacterial chemotaxis. SIAM J Appl Math. 1977;32(3):653-65. https://doi.org/10. $1137 / 0132054$

38. Alexandre G. Coupling metabolism and chemotaxis-dependent behaviours by energy taxis receptors. Microbiology. 2010;156:2283-93. https://doi.org/10.1099/mic.0.039214-0.

\section{Ready to submit your research? Choose BMC and benefit from:}

- fast, convenient online submission

- thorough peer review by experienced researchers in your field

- rapid publication on acceptance

- support for research data, including large and complex data types

- gold Open Access which fosters wider collaboration and increased citations

- maximum visibility for your research: over 100M website views per year

At $B M C$, research is always in progress.

Learn more biomedcentral.com/submissions 